\title{
Exploratory study describing 6 month outcomes for young children with autism who receive treatment as usual in Italy
}

This article was published in the following Dove Press journal:

Neuropsychiatric Disease and Treatment

8 April 2014

Number of times this article has been viewed

\author{
Filippo Muratori ${ }^{1,2}$ \\ Antonio Narzisi' \\ IDIA group
}

'Department of Developmental Neuroscience, IRCCS Stella Maris Foundation, Calambrone, Pisa, Italy; ${ }^{2}$ University of Pisa, Calambrone, Pisa, Italy
Correspondence: Filippo Muratori Department of Developmental Neuroscience, IRCCS Stella Maris Foundation, Via dei Giacinti, 2, I-560I8 Calambrone, Pisa, Italy Fax +395088 6247

Email filippo.muratori@fsm.unipi.it
Background: In the last few years, the results of different studies have confirmed, in different ways, the importance of early intervention for autism. This study aims to evaluate the role of early "as usual" interventions in the outcome of toddlers diagnosed with autism spectrum disorder (ASD).

Method: Seventy children with ASD aged between 24 and 48 months were recruited at different centers in Italy. They were evaluated by blind researchers at baseline and after 6 months of using Autism Diagnostic Observation Schedule-Generic (ADOS-G), Griffiths Mental Developmental Scales, and Vineland Adaptive Behavior scales. Parents filled out the MacArthur Inventory, Social Communication Questionnaire, and Child Behavior Check List. All children were referred to community providers for available interventions.

Results: At the endpoint, most of the children were still classified as having an ADOS-G classification of ASD. However, 21 (34.2\%) passed from autism to autism spectrum, and $3(4.2 \%)$ passed from autism spectrum to no spectrum. Treatment effects were obtained for cognitive functioning, language, adaptive behavior, and child behavior without differences between development-oriented and behavior-oriented interventions. Parent involvement was a mediator for the best clinical outcome. Baseline low impairments of communication, language comprehension, and gesture were predictors of positive outcome.

Conclusion: Treatment as usual, composed of individual therapy plus school-supported inclusion, may be an effective intervention in ASD. Better initial levels of communication in child and parent involvement during treatment have an important role for a positive outcome. Keywords: autism, preschoolers, treatment as usual, early intervention

\section{Introduction}

Early identification of autism spectrum disorders (ASD) has brought on the need for interventions that are significantly effective in reducing the effect of autism on child development. During the last 3 decades, the need for a treatment model that could be able to change the early natural history of the disorder has been addressed in a large number of studies, and plenty of literature exists. ${ }^{1-5}$

Historically, the first novel intervention developed was that of Lovaas, ${ }^{6}$ which in a 6 year follow-up showed better outcomes for children younger than 4 years with autism who were engaged in an intensive treatment compared with children in a less-intensive treatment or without any treatment. As reported by Rogers and Vismara, ${ }^{7}$ Lovaas' study has suggested a new vision of autism as a treatable disorder; however, its scientific validity has been repeatedly questioned by several authors. Among these, Schopler ${ }^{8}$ pointed out some critical points, such as the absence of outcome measures related to 
behavioral, social, and communicative skills; the imprecise criteria of intellectual levels for the selection of children; and the lack of precision in the allocation of subjects to the control group. To overcome these and other critical points, some criteria for assessing the methodological quality of studies on the effects of early treatments were defined. ${ }^{5-9}$ They include clinical diagnosis performed by independent evaluators according to international standards and supported by specific diagnostic tools such as the Autism Diagnostic Observation Schedule (ADOS) ${ }^{10}$ and Autism Diagnostic Interview ${ }^{\text {TM }}$, Revised; ${ }^{11}$ random assignment; outcome measures extended to both core aspects of autism and intellectual and adaptive functioning and conducted by independent examiners; and manualized treatment and measures for fidelity.

Different papers have taken into account these criteria, examining the effect of various interventions that can be located in the behavioral/developmental continuum. ${ }^{12} \mathrm{~A}$ wide systematic review ${ }^{13}$ of these studies suggested that four characteristics of early interventions in ASD could represent a solid foundation for efficacious interventions: parent involvement to support parental sensitivity to child cues; individualization to each infant's developmental profile; focus on a broad, rather than a narrow, range of learning targets; and temporal characteristics including beginning as early as the risk is detected, sufficient intensity, and an appropriately long duration. Some of these principles are addressed by nonmanualized treatments for children with ASD.

For example, in Italy, many young children are admitted to normal school with a personalized curriculum performed by a support teacher, they have psychomotor or speech therapy based on developmental profile, and some type of parent training is applied. Because young children with ASD have some type of interventions by local services, it has become important for new treatments to demonstrate what they really add to the effects of these interventions. In fact, more recent studies have compared new treatments versus the treatment as usual (TAU) that is available from local services. Green et $\mathrm{al}^{2}$ randomly assigned 152 children younger than 4 years with autistic disorder to the TAU plus PACT (Preschool Autism Communication Trial) intervention or to only TAU; at 1-year follow-up, in the PACT group, 30\% of children changed ADOS classification to autism spectrum, and 4 children $(5 \%)$ changed to no spectrum; however, even $24 \%$ of TAU children passed to autism spectrum classification, and 5 children $(7 \%)$ went out of the spectrum. Strauss et al, ${ }^{3}$ comparing children with ASD who underwent an early intensive behavioral intervention (EIBI) treatment with children who received TAU in Italy, described better effects of EIBI and moderate effects of
TAU in measures of autism severity and developmental and language skills. Oosterling et al, ${ }^{14}$ using a randomized control trial (RCT), evaluated the additional effects of nonintensive parent training focused on joint attention and language skills to TAU; no significant intervention effects were found. Carter ${ }^{15}$ compared Hanen's "More than Words" (HMTW), a parent-implemented intervention, with TAU; this RCT did not show the main effects of the HMTW intervention, but it pointed out treatment effects on child communication gains that were moderated by an initial lower object interest in the children. Pajareya ${ }^{16}$ designed a pilot study to test the efficacy of adding home-based developmental, individualdifference, relationship-based intervention to TAU. It was found that after the added home intervention at an average of 15 hours/week for 3 months, the intervention group made significantly greater gains on both functioning and autism level. Landa et $\mathrm{a}^{17}$ evaluated the effect of supplementing a comprehensive intervention with a specific curriculum targeting socially synchronous behaviors such as engaged imitation, joint attention, and affect sharing; a significant treatment effect was found for these behaviors, which were generalized to unfamiliar contexts and maintained through follow-up. Dawson ${ }^{1}$ compared the intensive Early Start Denver Model (ESDM) treatment with a less-intensive TAU, and at a 2-year follow-up, the ESDM group showed, compared with TAU, top scores in cognitive evaluation, verbal skills, and severity of autism. In particular, in the ESDM group, 7 (29.2\%) children passed from autistic disorder to spectrum compared with only one child in TAU.

Most of these recent articles on the efficacy of specific early interventions compared with or added to TAU have used an RCT scenario that is regarded as the "gold standard" of outcome research for empirically supported treatments. Nevertheless, implications of RCT have been much discussed. ${ }^{18,19}$ A central aspect of this discussion refers to the controversy between RCTs and naturalistic studies. Leichsenring ${ }^{20}$ has proposed that RCT and naturalistic studies refer to different domains of intended applications (laboratory versus field). This view has several important implications: naturalistic studies do not necessarily provide lower-level evidence than RCT, evidence from RCTs can have difficulties when transferred to practice in the field, and naturalistic studies are required to demonstrate that a type of intervention works in the field.

Thus, although the arrival of RCTs represents a substantial improvement for research on autism treatment, more recently the exclusive position of $\mathrm{RCT}$ as the method for demonstrating that a therapy works has been challenged, 
mainly because it is questionable whether the results of RCT are representative for clinical practice. ${ }^{18,19}$ Contrary to RCT, naturalistic studies are carried out under the conditions of clinical practice, ${ }^{21}$ but, paradoxically, they are not accepted as a method for demonstrating that a therapy works. ${ }^{22}$ The main argument against naturalistic studies concerns threats to the internal validity; that is, to the reduced possibility of controlling factors influencing outcome apart from treatment. However, naturalistic studies can support the external validity of the research; that is, whether results obtained from a small sample group can be extended to make predictions in a large sample.

Fernell ${ }^{4}$ conducted a naturalistic study on 208 ASD children, aged 20-54 months, and divided them into three cognitive subgroups: one with learning disability, one with developmental delay, and one with normal intellectual functioning. Data on type and intensity of intervention were gathered prospectively in a systematic fashion. Intervention was classified into intensive Applied Behavior Analysis (ABA) and nonintensive targeted interventions based on ABA principles. Vineland composite scores, as the primary outcome variable, increased during the 2-year period, and this increase was accounted for with the subgroup with normal cognitive functioning. However, there was no significant difference between the intensive and nonintensive groups. Individual variation was considerable, but no child in the study was "problem-free" at follow-up. Fernell ${ }^{4}$ concluded that ASD children do not benefit more from intensive ABA.

RCTs and naturalistic studies serve different complementary purposes and give answers to different questions of research. RCTs are required if a newly developed method of intervention is to be tested regarding its specific therapeutic effects; this is especially true if alternative treatments are already available. In contrast, the effectiveness of a treatment in the field could be better tested through a naturalistic study of high methodological quality. In any case, 40 years after the Lovaas era, when children with ASD were diagnosed later and were frequently institutionalized, both types of studies had to consider the effects of TAU on the children's outcome and the specific effects added by the new proposed treatments to a natural "treated" history of ASD. The role of TAU for early intervention in Europe is an ongoing topic explored by the Enhancing the Scientific Study of Early Autism-COST Action. ${ }^{23}$

Here we propose a naturalistic approach to early treatment to describe, for the first time in Italy, the role of TAU. To this aim, we used primary and secondary outcome measures in a multicenter, 6 month, longitudinal study.

\section{Materials and methods}

\section{Participants}

The study was performed at a total of 13 centers located in five Italian regions (Lombardia, Toscana, Emilia Romagna, Puglia, and Campania). Children's inclusion criteria were age between 24 months and 48 months; meeting criteria for autistic disorder or pervasive developmental disorder not otherwise specified according to the Diagnostic and Statistical Manual of Mental Disorders, 4th Edition, Text Revision, criteria; autism classification confirmed by the ADOS-G (administered from clinicians trained in the use of ADOS in a research setting); nonverbal development quotient of 50 or higher on the Griffiths Scales; ${ }^{24}$ it being the patient's first ASD diagnosis; and the patient being able to begin some type of intervention within 2 weeks of diagnosis. The whole sample consisted of 70 children ( 57 boys and 13 girls) with a mean age of 35.2 months (standard deviation, \pm 8.82 months; age range, 24-48 months). Forty-nine children underwent a Diagnostic and Statistical Manual of Mental Disorders, 4th Edition, Text Revision, diagnosis of autism, and 21 received a diagnosis of pervasive developmental disorder not otherwise specified. Fifty-eight children received an ADOS classification of autism, and 12 received an autism spectrum diagnosis. The mean nonverbal development quotient was 75.8 (range, 50-125), and the mean general quotient was 62.3 (range, 34-89).

\section{Measurements}

We used a multi-informant perspective on data collection by using professional observation or interview (ADOS-G and Griffiths Scales and Vineland Adaptive Behavior Scales II) ${ }^{25}$ and parent reports (MacArthur Communicative Development Inventories, Child Behavior Checklist 1 1/2-5, and Social Communication Questionnaire). ${ }^{26-28}$

The $\mathrm{ADOS}^{10}$ is an observation measure of current autism symptom severity; on the basis of language level, module one was used for all children. Only children with a classification of core autism or ASD on the ADOS algorithm were included in the final sample. We have used also the Calibrated Severity Scores (CSS), ${ }^{29}$ a standardized metric developed to assess core autism symptoms as a clinical entity distinct from cognitive and adaptive differences. This metric provides a means to assess symptoms of autism over time in a range between 1 and 10, where 1-3 account for no spectrum, 4-5 for ASD, and 6-10 for autism. In the original validation study, these scores were shown to be less influenced by verbal IQ, which accounted for $43 \%$ of variance in raw ADOS scores and only $10 \%$ in CSS scores. ${ }^{29}$ 
The Griffiths Mental Developmental Scales are a standardized developmental test for children from birth to 96 months of age. They comprise six scales, but because of the young age of the children, only five of the six subscales were administered: Locomotor, Personal-Social, Language, Eye and Hand Coordination, and Performance. Raw scores have been computed for each subscale and converted to general quotient scores, using tables of the analysis manual.

The Vineland Adaptive Behavior Scales, Second Edition (VABS-II), was administered as a parent interview and was used to assess the ability of children to perform the daily activities required for personal and social sufficiency. The VABS-II uses four specific domains: Communication, Daily Living Skills, Socialization, and Motor Skills. The subscale scores are added up to yield an adaptive behavior composite score.

The MacArthur Communicative Development Inventory (MCDI) was filled out by parents. It includes word comprehension (maximum score $=370$ ), word expression ( maximum score $=370$ ), and gestures ( maximum score $=60$ ). Because the children in the study were older than those in the normative groups, raw data were used instead of standard scores.

The Child Behavior Checklist 1 1/2-5 (CBCL) is a 100 item parent-report measure designed to record the behavioral peculiarities of preschoolers. Each item describes a specific behavior, and the parent is asked to rate its frequency on a three-point Likert scale. The scoring gives, among others, three main scores (Internalizing, Externalizing, and Total Problems). A T-score (for Internalizing and Externalizing, and for Total Problems) of 63 and above is considered clinically significant, and values between 60 and 63 identify a borderline clinical range; values beneath 60 are considered not clinical.

The Social Communication Questionnaire (SCQ) is filled out by parents to evaluate the communication skills and social functioning of children. SCQ provides a total score that can be interpreted in relation to specific cutoff points (a score higher than 15 is considered indicative of a risk for ASD). SCQ content parallels that of the Autism Diagnostic Interview $^{\mathrm{TM}}$, Revised, and the agreement between the two instruments is high and is substantially unaffected by age, sex, language, and performance IQ. ${ }^{30}$

\section{Procedures}

Two evaluations were performed by blind clinicians and by parents during the research project: at the beginning of treatment (T0) and at the treatment after 6 months (T1). An electronic grid has been realized and shared by the centers to register evaluations data and to report in detail the hours of treatment (school hours are included) and parent involvement during child/therapist work sessions. All centers filled out this grid and sent it to the Stella Maris Scientific Institute, the leader of the research project, where all data analyses were performed.

At $\mathrm{T} 0$, there were no significant differences among children of the different treatment centers regarding the mean scores of the assessment protocol.

The study was approved by the Istituto di Ricovero e Cura a Carattere Scientifico Stella Maris Foundation Research Ethics Committee, and at least one parent provided written consent.

\section{TAU}

Children received TAU for a mean of 11.2 hours per week without any economic charge for families. TAU is composed of specific treatments performed by child neuropsychiatric services (CNS) and of school inclusion with individual support teacher. In Italy, laws 517/1977 and 104/1992 established the right of inclusion for all children with disabilities, as well as some inclusion strategies, such as the right to be trained by a "support teacher" specifically trained to work in the field of disabilities (by means of a biennial special education course not specifically designed for ASD children). Before inclusion, children receive a "functional diagnosis" describing the child's strengths and weaknesses in all developmental domains; a "functional dynamic profile" prepared by the local CNS, together with teachers and parents, describing the expectations for the child's modification after a first period of inclusion; and an "individualized educational plan" describing the objectives of the supportive project. Regular and special teachers are supported by an interdisciplinary team provided by CNS. The inclusive Italian program is a coteaching model in which general and support teachers work together to teach students with and without disabilities in the same classroom.

Treatments offered by CNS include sessions of individual psychomotricity and/or speech and/or psycho-educative therapy. Each child's program includes individual goals and treatment objectives but is mainly based on staff expertise rather than manualized treatment protocols.

Treatments can be placed within a continuum ranging from highly structured behavioral approaches guided by a therapist in a nonnaturalistic setting to approaches that follow the interests of the child in a naturalistic setting and are based on a developmental curriculum in a relational-based context. 
Sometime, but not always, TAU includes hours of parent involvement, and some homework is provided to them. The parent involvement is intended as parent coaching that is a direct involvement of the parents during the child-therapist work sessions.

\section{Sample size}

A sample size a priori calculation was performed. Considering that in previous studies the percentage of ADOS-G classification improvement (that we considered as primary outcome) was around $30 \%,{ }^{1,2}$ we used a proportion difference of $30 \%$. Accordingly, the sample size was estimated as being composed of 68 subjects with an $\alpha$ error of 0.05 and a $\beta$ error of 0.10 .

\section{Data analysis}

All variables were examined for normality, using the Kolmogorov-Smirnov test; they were normally distributed.

Mean scores at intake and after 6 months of TAU were computed for all primary (ADOS scores and changes in diagnostic classification) and secondary (Griffiths, VABS-II, MCDI, CBCL, and SCQ) outcome measures. Student's $t$-test (two-tailed) with the estimate of effect sizes (Cohen's $d$ ) was carried out.

To determine whether a greater number of hours of treatment led to a better outcome, the ADOS-CSS was used and the Pearson partial correlation, covaried for age, was performed.

Student's $t$-test (two-tailed) with the estimate of effect sizes (Cohen's $d$ ) and multivariate analysis of variance, using Bonferroni adjustment, were carried out to find possible differences between parent involvement versus parents not involved and positive outcome (children who have improved their ADOS classification, passing from autism to spectrum or from spectrum to no spectrum) versus no positive outcome (children who do not have improved or have worsened their ADOS classification). Statistical analyses were performed using SPSS software version 20.0 (IBM Corporation, Armonk, NY, USA). An alpha level of 0.05 was used for all statistical analyses.

\section{Results}

Table 1 displays statistics for changes from $\mathrm{T} 0-\mathrm{T} 1$ on primary and secondary measures. Significant differences were found for all ADOS scores, on Griffiths Personal-Social and Hearing

Table I Child outcome after 6 months of treatment as usual

\begin{tabular}{|c|c|c|c|c|c|c|c|c|c|}
\hline & \multicolumn{2}{|c|}{ Baseline (T0) } & \multicolumn{3}{|c|}{ 6-month outcomes (TI) } & \multicolumn{3}{|c|}{ Student's t-test } & \multirow{2}{*}{$\begin{array}{l}\text { Effect size } \\
\text { Cohen's d }\end{array}$} \\
\hline & Mean & SD & Mean & SD & $\Delta$ & $t$ & df & P-value & \\
\hline \multicolumn{10}{|l|}{ Autism Diagnostic Observation Schedule } \\
\hline Total & 16.5 & 3.9 & 12.9 & 4.5 & -3.6 & 10.76 & 69 & 0.002 & 0.85 \\
\hline Communication & 5.7 & 1.6 & 4.6 & 2.1 & -1.1 & 4.99 & 69 & 0.029 & 0.58 \\
\hline Social Interaction & 10.7 & 2.8 & 8.2 & 2.9 & -2.5 & 10.41 & 69 & 0.002 & 0.87 \\
\hline Calibrated Severity Score & 7.1 & 2.0 & 6.0 & 1.8 & -1.01 & 5.02 & 69 & 0.028 & 0.57 \\
\hline \multicolumn{10}{|l|}{ Griffiths Mental Developmental Scales } \\
\hline Total scale (GQ) & 62.3 & 13.4 & 69.6 & 17.8 & 7.3 & 5.03 & 69 & 0.028 & 0.46 \\
\hline Locomotor development & 81.9 & 16.4 & 83.7 & 16.4 & 1.8 & 0.003 & 69 & 0.956 & 0.10 \\
\hline Personal-social development & 55.1 & 18.4 & 64.1 & 19.4 & 9 & 14.94 & 69 & 0.000 & 0.47 \\
\hline Hearing and speech & 37.4 & 17.4 & 51.1 & 26.3 & 13.7 & 13.40 & 69 & 0.001 & 0.87 \\
\hline Hand and eye coordination & 63.6 & 16.7 & 71.3 & 21.4 & 7.7 & 0.20 & 69 & 0.650 & 0.40 \\
\hline Performance test & 75.8 & 19.8 & 82.2 & 23.3 & 6.4 & 1.45 & 69 & 0.233 & 0.29 \\
\hline \multicolumn{10}{|c|}{ Vineland Adaptive Behavior Scales, Second Edition } \\
\hline Composite & 67.1 & 11.3 & 73.4 & 11.6 & 6.3 & 3.57 & 69 & 0.063 & 0.55 \\
\hline Communication & 59.8 & 14.4 & 69.1 & 15.0 & 9.3 & 1.07 & 69 & 0.304 & 0.63 \\
\hline Daily Living & 71.7 & 13.0 & 78.6 & 13.0 & 6.9 & 8.12 & 69 & 0.006 & 0.53 \\
\hline Socialization & 65.2 & 12.5 & 70.5 & 11.7 & 5.3 & 4.26 & 69 & 0.043 & 0.43 \\
\hline Motor & 81.5 & 14.0 & 85.2 & 13.7 & 3.7 & 0.05 & 69 & 0.813 & 0.26 \\
\hline \multicolumn{10}{|l|}{ MacArthur } \\
\hline Production & 58.5 & 93.3 & 131.8 & 131.2 & 73.3 & 2.87 & 69 & 0.095 & 0.64 \\
\hline Comprehension & 145 & 110.0 & 226.4 & 120.8 & 81.3 & 16.71 & 69 & 0.000 & 0.70 \\
\hline Gestures & 29.8 & 14.4 & 42.3 & 12.8 & 12.5 & 18.80 & 69 & 0.000 & 0.91 \\
\hline \multicolumn{10}{|l|}{ Child Behavior Checklist I $1 / 2-5$} \\
\hline Internalizing problems & 63.5 & 11.6 & 59.5 & 9.7 & -4 & 11.64 & 69 & 0.001 & 0.37 \\
\hline Externalizing problems & 57.6 & 12.3 & 52.9 & 9.6 & -4.7 & $|1.8|$ & 69 & 0.001 & 0.42 \\
\hline Total problems & 62.3 & 13.7 & 57.0 & 10.9 & -5.3 & 13.27 & 69 & 0.001 & 0.42 \\
\hline Social Communication Questionnaire & 17.6 & 5.5 & 14.0 & 5.6 & -3.6 & 25.12 & 69 & 0.000 & 0.64 \\
\hline
\end{tabular}

Abbreviations: SD, standard deviation; df, degree of freedom; GQ, general quotient. 
and Speech, and on VABS-II Daily Living and Socialization subscales. MCDI shows an improved score of 81.3 points on language comprehension and of 12.8 on gestures. Significant differences were revealed on CBCL scales $(P=0.001)$ and SCQ scores $(P<0.001)$.

\section{Autism severity and hours of treatment}

The ADOS-CSS difference score (T1-T0) did not correlate with the number of hours of treatment $(r=0.047 ; P=0.868)$.

\section{Parent's involvement}

Thirty-six children (31 boys and 5 girls) out of 70 had their parents involved in the treatment; this group was compared with the 34 ( 28 boys, 6 girls) children whose parents were not actively involved in treatment. At T0, a significant difference between the two groups was present as far as the age of children is concerned (parents involved, 27.9 \pm 2.62 months; parents not involved, $40.2 \pm 5.25$ months; $t[69]=3.11 ; P=0.001$ ). At T1, the multivariate analysis of variance (group $\times$ time), covaried for age and hours of treatment, showed significant differences on the ADOS Total scores (parents involved, T0: 17.8 \pm 3.8 , and T1: 12.6 \pm 3.7 ; parents not involved, T0: $16 \pm 3.9$ and T1: 13.2 \pm 5.2 ; $F[1,68]=4.21 ; P=0.017)$. Children with parents involved in treatment had significantly higher improvements compared with the group of children whose parents were not involved.

\section{Positive outcome}

Forty-five (64\%) of 70 children maintained the same ADOS classification (37 continued to be classified as autistic and eight remained in the autism spectrum). ADOS classification changed for $21(34.2 \%)$ children from autism to autism spectrum and for three (4.2\%) from autism spectrum to no spectrum. In one case, the diagnosis changed from the spectrum to autism. The flow chart in Figure 1 shows 70 children who changed or did not change their allocation either within the spectrum or outside the spectrum. At T0, significant differences between the 24 children with positive outcome (PO) and all others were present on ADOS Communication (PO, 5.09 \pm 1.08 versus other, 6.13 \pm 1.83 ; $t[69]=2.50 ; P=0.015$; Cohen's $d=0.069)$, Comprehension (PO, 177.9 \pm 98.03 versus other, 123.9 $\pm 108.1 ; t[69]=-2$; $P=0.049$; Cohen's $d=0.052$ ), and Gestures (PO, 34.6 \pm 11.5 versus other, $27.1 \pm 15.1 ; t[69]=-2.09 ; P=0.040$; Cohen's $d=0.052$ ) at MCDI.

\section{Discussion}

Here we present longitudinal changes of children with autism during the first 6 months of treatment immediately after first diagnosis. Results are a contribution to the research field on early treatment of children younger than 4 years with ASD in Italy. The naturalistic approach of our study is its strength: Cases arrived at clinics spontaneously, and they were representative of a real Italian population of young children who received usual treatments from their local services. Nevertheless, it has the weaknesses of an inaccurate identification and manualization of the applied treatment strategies. Hence, we are unable to make any hypothesis regarding which specific treatment ingredient is more useful

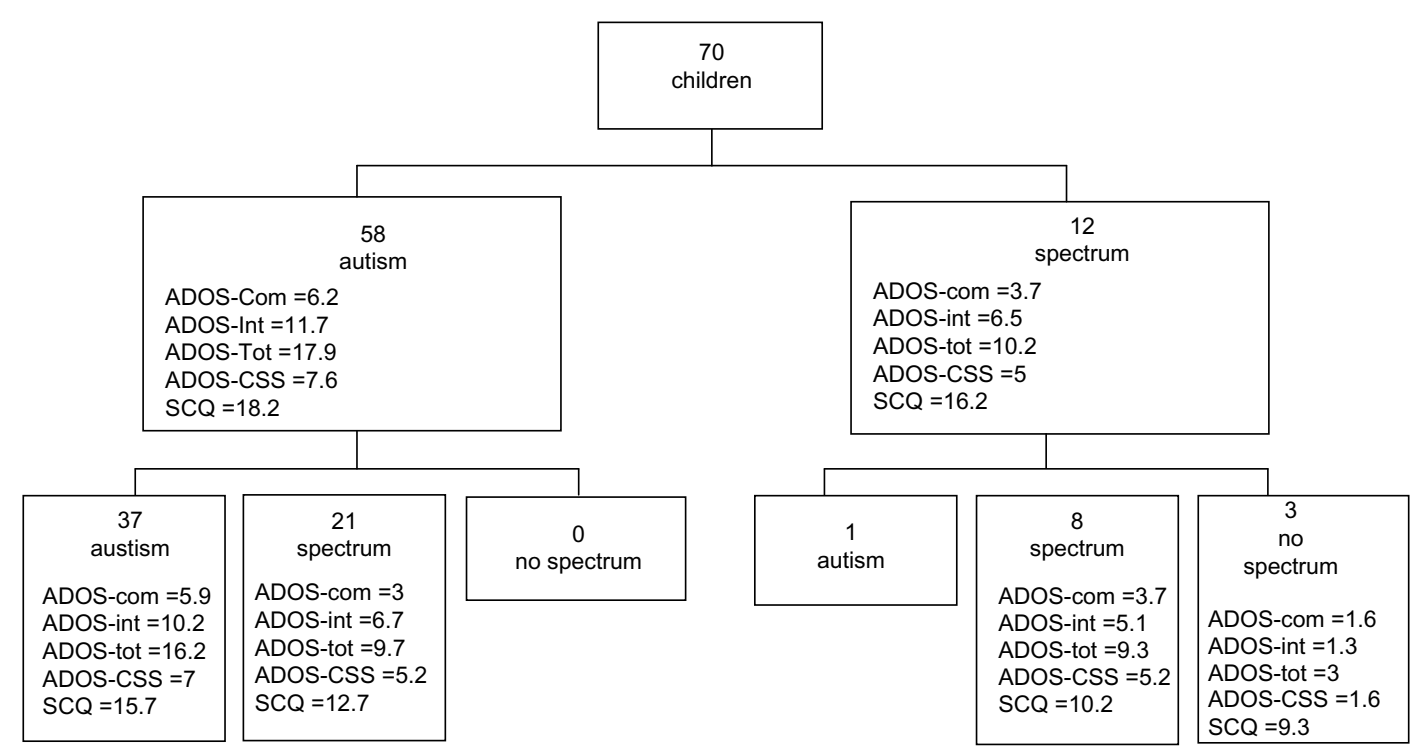

Figure I Changes on Autism Diagnostic Observation Schedule classification from T0 (baseline) to TI (6-month follow-up).

Abbreviations: ADOS, Autism Diagnostic Observation Schedule; Com, communication; Int, social interaction; Tot, total; CSS, Calibrated Severity Scores; SCQ, Social Communication Questionnaire. 
for a better outcome. For example, we did not have enough information to make a contribution to the field with regard to the role of psychomotricity (or play therapy) during this early age, which is an important topic, as motor dysfunctions are increasingly described in ASD. ${ }^{31}$ In the same way, hours of school support and hours of formal treatment were melded, so we cannot distinguish specific effects of these two components of TAU. In the future, a comparison between the effect of a similar number of hours of school support and of formal treatment in two groups of children with ASD could improve our understanding of the effects of singular ingredients of TAU.

In general, during the first 6 months of treatment, significant modifications occur in terms of autism severity and cognitive and linguistic development, as well as in adaptive behavior and comorbid psychopathology.

Although the lack of a RCT design meant that it is difficult to say whether TAU had any effect above no treatment, the implications of these modifications are manifold. First, they emphasize that the 6 month assessments of young diagnosed children could be an optimal timeframe for the reassessments; as during this short period, clinical modifications are so diffusive that for many cases, it is necessary to revise the diagnosis and check the objectives of treatment.

Second, our results identify early autism not as a rigid and stable disorder within the first 6 months of treatment. Instead, ADOS improvements and the parallel significant reduction on SCQ describe early autism as a disorder with a certain degree of instability. On this subject, a wide debate is ongoing. ${ }^{32,33}$ Turner and Stone ${ }^{34}$ indicated a stability of diagnosis in only $65 \%$ of children diagnosed before the age of 3 years; in contrast, Macari et $\mathrm{al}^{35}$ showed a higher diagnosis stability. A recent systematic ${ }^{36}$ review on diagnostic stability points out that the overall diagnostic stability is $85 \%-90 \%$ for autism disorder, whereas for other ASD, it is $14 \%-61 \%$; our data match with the conclusion of this review that underlines that the broader diagnosis of other ASDs (not including autism) is not a stable diagnostic category.

Third, clinical changes are observed in many other domains. Of importance are changes on the CBCL, an instrument that is rarely used as an outcome tool in autism. CBCL changes can be read in two complementary ways: on the one hand, the reduction of internalizing problems is a further confirmation of the improvement of autistic symptoms; in fact, different studies ${ }^{37,38}$ have described this cluster as a specific expression of autistic symptomatology. On the other hand, a reduction in CBCL Total score might indicate a generally lower propensity to psychiatric comorbidity that is frequently reported in $\mathrm{ASD}^{39}$ and that could worsen the clinical picture of autism.

Fourth, our research can contribute to the question of whether longitudinal changes are related to a specific treatment or whether they are part of the natural evolution of ASD, particularly when it is diagnosed and treated at an early age. ${ }^{40,41}$ All five longitudinal studies ${ }^{32-34,40,41}$ that measured early intervention as a possible predictor of diagnostic stability did not find any difference, as far as treatments are regarded, between children who maintained their diagnosis over time and those who did not. Because the inclusion of a control group without any treatment is virtually impossible for obvious ethical reasons, our study suggests that future efficacy studies should consider TAU as a control group and be precise about what the new treatment adds to the naturalistic outcome of TAU children.

Fifth, our research has enabled us to investigate the role of parent involvement during early treatment. We have been able to identify within our sample a group of children who have benefited from the active involvement of parents and to compare their outcome with that of a group of children whose parents were not actively involved. This comparison revealed significant differences on most of the outcome measures in favor of the group in which the parents were involved. This result, although expected, is a confirmation of the importance of involving parents, who, because of being present in the therapy room, are likely to acquire new knowledge on their child and to reduce the stress of having a child with special needs. ${ }^{13}$ We can hypothesize that the increase of knowledge and the decrease of stress allow ongoing parent-child interaction to become an active part of treatment that is applied in everyday life. The positive effect of parent involvement is also promising in relation to the renewed interest in parent-child interactions as a possible outcome measure. ${ }^{42}$ Until now, efficacy studies have left aside the thorough investigation of treatment effects on parent-child interaction, which is, in fact, needed for the centrality that has been attributed to parent-mediated treatment by different national guidelines. ${ }^{43}$

Finally, because our study does not differ in methodology (with regard to outcome protocol) from other effectiveness studies, it is possible to compare changes in our sample with those of samples described in other studies. ${ }^{1,2,44}$ In particular, we propose some considerations regarding changes on ADOS classification. About $34 \%$ of our children show an improvement from autism to autism spectrum, and $4 \%$ fall off the autism spectrum; these percentages of improvement are similar to the percentage of true responders found in 
other effectiveness studies. The fact that similar changes are obtained by our sample after 6 months of treatment and by samples in these studies after 12 months of treatment might suggest that the most salient changes happen during the first part of treatment and that they are followed by a slower improvement. ${ }^{45}$ The similar percentage of PO could also suggest the presence, inside autism classification, of children who are more responsive to treatment. ${ }^{46}$ In the future it will be of seminal importance to know whether these ASD children are of the same category in various studies or whether they are different children affected by a different type of autism that responds differently to specific elements of each treatment. Schreibman ${ }^{47}$ has underlined the need for developing a "technology" for the individualization of treatment through the exploitation, at the same time or in a sequential way, of different treatments among the many that are currently available for children with autism. ${ }^{12}$ Second, the extraction of this group of children with a PO could allow the identification of factors that can help clinicians in tailoring treatment and prognosis for this minority of children who are candidates for a PO. Among factors affecting PO, cognitive development was not found; as expected, PO correlated with a less-severe autism on ADOS. This result is in agreement with that of Vivanti, ${ }^{48}$ who, in a longitudinal study of early autism, has proposed that low cognitive level, frequently observed in autism, is not a comorbid feature of autism but is a consequence of the severity of social-communication deficits on the experience-dependent mechanisms underlying neurocognitive development. In the future, the identification of predictors of PO could help us examine the question of what works for whom with regard to autism. Carter ${ }^{15}$ has identified the low rate of baseline object interest as a predictor for good responsivity to her HMTW treatment and concludes that children who evidence higher initial object interest may require different strategies than those provided by the HMTW curriculum.

\section{Clinical implications}

Notwithstanding the need of a longer longitudinal assessment to better clarify the clinical outcome of early autism and the effective role of therapeutic factors, and to exclude the observer effect (also referred to as the Hawthorn effect), our results may indicate some useful key points in communicating diagnosis and starting treatment when we are faced with parents who have received the first diagnosis of autism for their child. First, we can reasonably return to parents a working diagnosis ${ }^{49}$ considering PO as a real chance. Second, when we start treatment, it is mandatory that we consider the active involvement of parents as an element of seminal importance for early treatment. Third, it is necessary to develop a personalized treatment that takes into consideration the unique child profile and the more useful ingredients for that child among currently available and effective treatments. Fourth, the observed changes make it absolutely mandatory that there be a regular reassessment of each child, at least every 6 months. It is in fact through these assessments that we can more accurately verify the effectiveness of the proposed treatment and possibly modify or augment it if clearly not effective. ${ }^{46}$ Finally, we cannot underscore the importance of inclusion in the outcome of our children. Through inclusive education, our children spent most of their time at school, where they received specialized education and continuous confrontation with the normal behaviors of their peers. We cannot exclude the fact that, in part, the outcome of our children was influenced by this beyond the specific treatments provided by the services. ${ }^{50}$ As suggested by Parsons ${ }^{51}$ our research indicates the compelling need to conduct better educational research through collaborative partnerships between researchers and practitioners; we think this type of research could improve our understanding of outcomes for young children in the spectrum.

\section{Acknowledgment}

This study has been financially supported by the Italian Ministry of Health (Strategic Program IDIA "Inquiry into Disruption of Intersubjective Equipment in Autism Spectrum Disorder in Childhood").

The IDIA group is composed of $\mathrm{MD}$ and $\mathrm{PhD}$ allocated in different Child Neuropsychiatric Services (CNS) in the north, center, and south of Italy: Tancredi Raffaella, Massagli Angelo, Esposito Dalila, Villa Laura, Valli Angela, Molteni Massimo, Militerni Roberto, Frolli Alessandro, Balottin Umberto, Carigi Tiziana, Gallo Maria Linda, Giuberti Virginia, Pitanti Antonella, Germelli Chiara, Giorgi Antonella, Vannucchi Lucia, Armellini Marco, Campanile Sara, Casella Cristina, Monti Annalisa, Masini Silvia, Pini Giorgio, Calugi Simona.

\section{Disclosure}

The authors report no conflicts of interest in this work.

\section{References}

1. Dawson G, Rogers S, Munson J, et al. Randomized, controlled trial of an intervention for toddlers with autism: the Early Start Denver Model. Pediatrics. 2010;125(1):e17-e23.

2. Green J, Charman T, McConachie H, et al; PACT Consortium. Parent-mediated communication-focused treatment in children with autism (PACT): a randomised controlled trial. Lancet. 2010;375(9732): $2152-2160$. 
3. Strauss K, Vicari S, Valeri G, D'Elia L, Arima S, Fava L. Parent inclusion in Early Intensive Behavioral Intervention: the influence of parental stress, parent treatment fidelity and parent-mediated generalization of behavior targets on child outcomes. Res Dev Disabil. 2012;33(2): 688-703.

4. Fernell E, Hedvall $\AA$, Westerlund J, et al. Early intervention in 208 Swedish preschoolers with autism spectrum disorder. A prospective naturalistic study. Res Dev Disabil. 2011;32(6):2092-2101.

5. Rogers SJ, Estes A, Lord C, et al. Effects of a brief Early Start Denver model (ESDM)-based parent intervention on toddlers at risk for autism spectrum disorders: a randomized controlled trial. J Am Acad Child Adolesc Psychiatry. 2012;51(10):1052-1065.

6. Lovaas OI. Behavioral treatment and normal educational and intellectual functioning in young autistic children. J Consult Clin Psychol. 1987;55(1):3-9.

7. Rogers SJ, Vismara LA. Evidence-based comprehensive treatments for early autism. J Clin Child Adolesc Psychol. 2008;37(1):8-38.

8. Schopler E, Short A, Mesibov G. Relation of behavioral treatment to "normal functioning": comment on Lovaas. J Consult Clin Psychol. 1989;57(1):162-164.

9. Eikeseth S. Outcome of comprehensive psycho-educational interventions for young children with autism. Res Dev Disabil. 2009;30(1): $158-178$.

10. Lord C, Risi S, Lambrecht L, et al. The Autism Diagnostic Observation Schedule-Generic: a standard measure of social and communication deficits associated with the spectrum of autism. J Autism Dev Disord. 2000;30(3):205-223.

11. Lord C, Rutter M, Le Couteur A. Autism Diagnostic Interview-Revised: a revised version of a diagnostic interview for caregivers of individuals with possible pervasive developmental disorders. JAutism Dev Disord. 1994;24(5):659-685.

12. Ospina MB, Krebs Seida J, Clark B, et al. Behavioural and developmental interventions for autism spectrum disorder: a clinical systematic review. PLoS One. 2003(11):e3755.

13. Wallace KS, Rogers SJ. Intervening in infancy: implications for autism spectrum disorders. J Child Psychol Psychiatry. 2010;51(12): 1300-1320.

14. Oosterling I, Visser J, Swinkels S, et al. Randomized controlled trial of the focus parent training for toddlers with autism: 1-year outcome. J Autism Dev Disord. 2010;40(12):1447-1458.

15. Carter AS, Messinger DS, Stone WL, Celimli S, Nahmias AS, Yoder P. A randomized controlled trial of Hanen's 'More Than Words' in toddlers with early autism symptoms. J Child Psychol Psychiatry. 2011;52(7):741-752.

16. Pajareya K, Nopmaneejumruslers K. A pilot randomized controlled trial of DIR/Floortime ${ }^{\mathrm{TM}}$ parent training intervention for pre-school children with autistic spectrum disorders. Autism. 2011;15(5):563-577.

17. Landa RJ, Holman KC, O'Neill AH, Stuart EA. Intervention targeting development of socially synchronous engagement in toddlers with autism spectrum disorder: a randomized controlled trial. JChild Psychol Psychiatry. 2011;52(1):13-21.

18. Beutler LE. Identifying empirically supported treatments: what if we didn't? J Consult Clin Psychol. 1998;66(1):113-120.

19. Fonagy P. Process and outcome in mental health care delivery: a model approach to treatment evaluation. Bull Menninger Clin. 1999;63(3) 288-304.

20. Leichsenring F. Randomized controlled versus naturalistic studies: a new research agenda. Bull Menninger Clin. 2004;68(2):137-151.

21. Shadish WR, Matt GE, Navarro AM, Phillips G. The effects of psychological therapies under clinically representative conditions: a meta-analysis. Psychol Bull. 2000;126(4):512-529.

22. Chambless DL, Ollendick TH. Empirically supported psychological interventions: controversies and evidence. Annu Rev Psychol. 20052:685-716.

23. ESSEA. Enhancing the scientific study of early autism. [homepage on the Internet]. Available from http://www.cost-essea.com/. Accessed March 27, 2014.
24. Griffiths R. The Abilities of Young Children: A Comprehensive System of Mental Measurement for the First Eight Years of Life. Bucks, United Kingdom: The Test Agency Limited; 1984.

25. Sparrow S, Balla D, Cicchetti D. The Vineland Adaptive Behavior Scales: Interview Edition. Circle Pines, MN: American Guidance Service; 1984.

26. Fenson L, Pethick S, Renda C, Cox JL, Dale PS, Reznick JS. Short-form versions of the MacArthur Communicative Developmental Inventories. Appl Psycholinguistics. 2000;21:95-116.

27. Achenbach TM, Rescorla L. Manual for the ASEBA Preschool Forms and Profile. Burlington, VT: Univeristy of Vermont; 2000.

28. Rutter M, Bailey A, Lord C. Social Communication Questionnaire (SCQ) Manual. Los Angeles, CA: Western Psychological Services; 2000.

29. Gotham K, Pickles A, Lord C. Standardizing ADOS scores for a measure of severity in autism spectrum disorders. JAutism Dev Disord. 2009;39(5):693-705.

30. Corsello C, Hus V, Pickles A, et al. Between a ROC and a hard place: decision making and making decisions about using the SCQ. J Child Psychol Psychiatry. 2007;48(9):932-940.

31. Esposito M, Gimigliano F, Ruberto M, et al. Psychomotor approach in children affected by nonretentive fecal soiling (FNRFS): a new rehabilitative purpose. Neuropsychiatr Dis Treat. 2019:1433-1441.

32. Jónsdóttir SL, Saemundsen E, Asmundsdóttir G, et al. Follow-up of children diagnosed with pervasive developmental disorders: stability and change during the preschool years. J Autism Dev Disord. 2007;37(7): 1361-1374.

33. Chawarska K, Klin A, Paul R, Macari S, Volkmar F. A prospective study of toddlers with ASD: short-term diagnostic and cognitive outcomes. $J$ Child Psychol Psychiatry. 2009;50(10):1235-1245.

34. Turner LM, Stone WL. Variability in outcome for children with an ASD diagnosis at age 2. J Child Psychol Psychiatry. 2007;48(8): 793-802.

35. Macari SL, Campbell D, Gengoux GW, Saulnier CA, Klin AJ, Chawarska K. Predicting developmental status from 12 to 24 months in infants at risk for Autism Spectrum Disorder: a preliminary report. J Autism Dev Disord. 2012;42(12):2636-2647.

36. Woolfenden S, Sarkozy V, Ridley G, Coory M, Williams K. A systematic review of two outcomes in autism spectrum disorder - epilepsy and mortality. Dev Med Child Neurol. 2012;54(4):306-312.

37. Muratori F, Narzisi A, Tancredi R, et al. The CBCL $1.5-5$ and the identification of preschoolers with autism in Italy. Epidemiol Psychiatr Sci. 2011;20(4):329-338.

38. Narzisi A, Calderoni S, Maestro S, Calugi S, Mottes E, Muratori F. Child

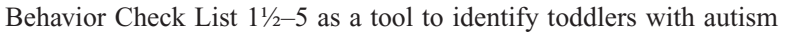
spectrum disorders: a case-control study. Res Dev Disabil. 2013;34(4): 1179-1189.

39. Kohane IS, McMurry A, Weber G, et al. The co-morbidity burden of children and young adults with autism spectrum disorders. PLoS One. 2017;(4):e33224.

40. Eaves LC, Ho HH. The very early identification of autism: outcome to age 4 1/2-5. J Autism Dev Disord. 2004;34(4):367-378.

41. Lord C, Risi S, DiLavore PS, Shulman C, Thurm A, Pickles A. Autism from 2 to 9 years of age. Arch Gen Psychiatry. 2006;63(6): 694-701.

42. Vernon TW, Koegel RL, Dauterman H, Stolen K. An early social engagement intervention for young children with autism and their parents. $J$ Autism Dev Disord. 2012;42(12):2702-2717.

43. Oono IP, Honey EJ, McConachie H. Parent-mediated early intervention for young children with autism spectrum disorders (ASD). Cochrane Database Syst Rev. 30, 2013;4:CD009774.

44. Smith T, Groen AD, Wynn JW. Randomized trial of intensive early intervention for children with pervasive developmental disorder. $\mathrm{Am} \mathrm{J}$ Ment Retard. 2000;105(4):269-285.

45. Vivanti G, Manzi B, Benvenuto A, Battan B, Curatolo P. An Italian Prospective Study on Autism Treatment: The Earlier, the Better? Autism Open Access. 2011;1:102. 
46. Dawson G, Bernier R, Ring RH. Social attention: a possible early indicator of efficacy in autism clinical trials. J Neurodev Disord. 2012;4(1):11.

47. Stahmer AC, Schreibman L, Cunningham AB. Toward a technology of treatment individualization for young children with autism spectrum disorders. Brain Res. 22, 2011;1380:229-239.

48. Vivanti G, Barbaro J, Hudry K, Dissanayake C, Prior M. Intellectual development in autism spectrum disorders: new insights from longitudinal studies. Front Hum Neurosci. 2013;7:354.
49. Charman T. What does the term 'working diagnosis' mean? J Autism Dev Disord. 2005;35(4):539-540.

50. Fountain C, Winter AS, Bearman PS. Six developmental trajectories characterize children with autism. Pediatrics. 2012;129(5): e1112-e1120.

51. Parsons S, Charman T, Faulkner R, Ragan J, Wallace S, Wittemeyer K. Commentary - Bridging the research and practice gap in autism: the importance of creating research partnerships with schools. Autism. 2013;17(3):268-280.

\section{Publish your work in this journal}

Neuropsychiatric Disease and Treatment is an international, peerreviewed journal of clinical therapeutics and pharmacology focusing on concise rapid reporting of clinical or pre-clinical studies on a range of neuropsychiatric and neurological disorders. This journal is indexed on PubMed Central, the 'PsycINFO' database and CAS.
The manuscript management system is completely online and includes a very quick and fair peer-review system, which is all easy to use. Visit http://www.dovepress.com/testimonials.php to read real quotes from published authors.

Submit your manuscript here: http://www.dovepress.com/neuropsychiatric-disease-and-treatment-journal 